\title{
The Minimal Primal Ideal Space of a Separable C*-Algebra
}

\author{
FERDINAND BECKHOFF
}

\section{Introduction}

In this paper, the device of the minimal primal ideal space of a $C^{*}$-algebra is used to establish a linearity result for quasistates and quasitraces on a class of separable liminal $C^{*}$-algebras. This can be used to describe all lower semicontinuous dimension functions on such algebras by Borel measures on their Jacobson spectrum. In another application it is shown that a separable liminal unital $C^{*}$-algebra can be represented by Borel measurable sections living on the Gelfand space of its center where the fibres are quotients by minimal primal ideals.

Let $Q$ be a $C^{*}$-algebra. On $\operatorname{Id}(Q)$, the set of closed two-sided ideals of $Q$, we have at least two important topologies.

(1) The weak topology $\tau_{w}$. This topology is generated by the sets of the form $U_{J}=\{I \in \operatorname{Id}(\mathscr{Q}) \mid I \not \supset J\}$, where $J$ runs through $\operatorname{Id}(\mathscr{Q})$. In general this topology is not Hausdorff. The restriction of $\tau_{w}$ to $\operatorname{Prim}(\mathcal{Q})$, the space of primitive ideals, coincides with the Jacobson topology. The set of primal ideals, denoted by $\operatorname{Primal}(\mathscr{Q})$, then is the $\tau_{w}$-closure of $\operatorname{Prim}(\mathscr{Q})$ in $\operatorname{Id}(\mathscr{Q})$. Let $\operatorname{Pl}(Q)$ be Primal $(Q)$ without the trivial primal ideal $Q$. An application of Zorn's lemma shows that each primal ideal contains a minimal primal ideal. Let $\mathrm{MP}(\mathfrak{Q})$ denote the space of all minimal primal ideals. A good reference for all this is [3].

(2) The strong topology or Fell topology $\tau_{s}$. This can be defined as the weakest topology making all the maps $\phi_{x}: \operatorname{Id}(\mathfrak{Q}) \rightarrow \mathbf{R}, \phi_{x}(I)=\|x+I\|$, continuous where $x$ runs through $Q$. This topology has been introduced by Fell [16] by describing a topological base; it has also been investigated in [13]. It is finer than $\tau_{w}$ and Hausdorff. $\tau_{w}$ and $\tau_{s}$ coincide when restricted to $\operatorname{MP}(\mathbb{Q})$ [3, Cor. 4.3].

These ideal spaces will be used in this paper to obtain results about the linearity of continuous quasistates on certain $\mathrm{C}^{*}$-algebras and about a representation of certain $C^{*}$-algebras by Borel sections living on the Gelfand space $T$ of $\operatorname{cen}(Q)$, the center of $Q$.

The main device is the map $\Phi: \operatorname{Pl}(Q) \rightarrow T_{\infty}$, which we now define. $T_{\infty}$ is the one-point compacitification of $T$. For $a \in \operatorname{cen}(\mathscr{Q})$ let $\hat{a}: \operatorname{Prim}(\mathscr{Q}) \rightarrow C$ be the 
Dauns-Hofmann transformation of $a$; that is, $\hat{a}(\operatorname{ker}(\pi))=\pi(a)$, which is a scalar operator. This is a continuous map vanishing at infinity if Prim( $(\mathbb{Q})$ carries the topology $\tau_{w}[12,3.3 .7]$. For any $P \in \operatorname{Prim}(\mathscr{Q})$, the map $a \mapsto \hat{a}(P)$ is a homomorphism on cen $(\mathscr{Q})$, and hence determines a unique point $t \in T_{\infty}$ such that $\hat{a}(P)=a(t)$, where the commutative $C^{*}$-algebra cen $(Q)$ is identified with its Gelfand representation. This $t$ will be denoted by $\Phi(P)$. By [3, Prop. 3.8] all continuous bounded maps $f: \operatorname{Prim}(Q) \rightarrow \mathbf{C}$ may be uniquely extended to bounded continuous maps $\bar{f}: \mathrm{Pl}(Q) \rightarrow \mathbf{C}$, where $\bar{f}(I):=f(P)$ if $I \subset P \in \operatorname{Prim}(\mathfrak{Q})$. So we may extend $\Phi$ to a map $\mathrm{Pl}(\mathfrak{Q}) \rightarrow T_{\infty}$, which will also be denoted by $\Phi$.

If $I_{\alpha}$ converges to $I$ in $\left(\mathrm{Pl}(\mathfrak{Q}), \tau_{w}\right)$, then for any $a \in \operatorname{cen}(\mathfrak{Q})$ we have

$$
a\left(\Phi\left(I_{\alpha}\right)\right)=\overline{\hat{a}}\left(I_{\alpha}\right) \rightarrow \overline{\hat{a}}(I)=a(\Phi(I)),
$$

and so $\Phi\left(I_{\alpha}\right) \rightarrow \Phi(I)$ by the definition of the Gelfand topology. So $\Phi$ is continuous.

An element $t \in T$ defines a pure state on $\operatorname{cen}(Q)$ which can be extended to a pure state on $Q$, giving an irreducible representation and hence a primitive ideal $P$. Obviously $\Phi(P)=t$. If $Q$ is quasicentral (i.e., no primitive ideal contains the center), then obviously the zero homomorphism is not in the image of $\Phi$ and therefore $\Phi(\operatorname{Pl}(Q))=T$. In the non-quasicentral case we will have $\Phi(\operatorname{PI}(Q))=T_{\infty}$.

By the definition of $\Phi$ on $\operatorname{Pl}(\mathscr{Q})$ it is easy to see that already $\Phi(\operatorname{MP}(Q)) \supset$ $T$. In general $\Phi$ is neither injective nor open.

The above-mentioned results will be achieved by considering certain crosssections $T \rightarrow \mathrm{MP}(Q)$ of $\Phi$. The next section is devoted to an important property of these cross-sections. Applications will be given in the subsequent sections.

\section{On a Theorem of Dixmier; Cross-Sections}

In [14] Dixmier proved the following theorem: Let $Q$ be a separable and liminal $C^{*}$-algebra such that $\operatorname{Prim}(\mathfrak{Q})$ is compact. Then the interior of the set of separated points is dense in Prim(Q). First this theorem will be generalized slightly, and then some conclusions concerning cross-sections of the map $\Phi: \operatorname{MP}(\mathscr{Q}) \rightarrow T_{\infty}$ will be drawn.

THEOREM 2.1. Let $\mathbb{Q}$ be a separable liminal and quasicentral $C^{*}$-algebra. Then the interior of the set of separated points is dense in $\operatorname{Prim}(Q)$.

Proof. From the proof given in [14] we conclude that all open subsets which are Hausdorff in their relative topology are $F_{\sigma}$-sets in Prim $(\mathscr{Q})$ if $\mathscr{Q}$ is separable and liminal and Prim(Q) compact. Now let $\left(u_{n}\right)_{n}$ be a countable approximate unit in cen $(Q)$, which is guaranteed by [4]. Then it is easy to conclude that Prim $(Q)$ is a countable union of closed and compact sets; indeed the $\left\{P \in \operatorname{Prim}(\mathscr{Q}) \mid \widehat{u}_{n}(P) \geq 1 / m\right\}$ may be used. So we can apply the argument 
of Dixmier to these sets separately and obtain that all open Hausdorff sets of Prim $(Q)$ are $F_{\sigma}$. Then go back to the proof given in [14] to establish the claimed result.

Recall that an ideal of a $C^{*}$-algebra is said to be essential if the corresponding open set in Prim $(\mathfrak{Q})$ is dense in the Jacobson topology.

THEOREM 2.2. Let $Q$ be a $C^{*}$-algebra possessing an essential separable liminal and quasicentral ideal. Let $\phi: T \rightarrow \mathrm{MP}(Q)$ be any cross-section of $\Phi$ : $\operatorname{MP}(\mathfrak{Q}) \rightarrow T_{\infty}$. Then $\phi(T)$ is dense in $\mathrm{MP}(\mathfrak{Q})$.

Proof. Let $J$ be the ideal in the assumption about $\mathcal{Q}$, and let $U$ be the interior of the sets of separated points in $\operatorname{Prim}(J)$. Since $J$ is essential and by the theorem above, we conclude that $U$ is $\tau_{w}$-dense in $\operatorname{Prim}(\mathscr{Q})$. Clearly $\overline{\operatorname{MP}(Q)})^{\tau_{w}}=\overline{\operatorname{Prim}(Q)}^{\tau_{w}}$, and so the result will follow from $U \subset \phi(T)$.

(i) First let us prove $U \subset \operatorname{MP}(\mathfrak{Q})$. Let $P \in U, Q \in \operatorname{Prim}(\mathfrak{Q})$, and let $\left(P_{\alpha}\right)_{\alpha}$ be a net in $\operatorname{Prim}(Q)$ converging to $P$ and $Q$. By [5, Prop. 3.2] it is enough to prove $P=Q$. Assume $Q \notin \operatorname{Prim}(J)$, that is, $Q \supset J$. Since $J$ is quasicentral, there is $a \in \operatorname{cen}(J)=\operatorname{cen}(Q) \cap J$ such that $\hat{a}(P)=1$. Then $1=\hat{a}(P)=$ $\lim _{\alpha} \hat{a}\left(P_{\alpha}\right)=\hat{a}(Q)=0$, so $Q$ must be in $\operatorname{Prim}(J)$. If $Q \not \supset P$ then $P$ and $Q$ are separated, which is impossible because they belong to a common limit set. So by liminality of $J$ we conclude $P=Q$.

(ii) If $V \subset U$ is $\tau_{w}$-compact, then $V$ is $\tau_{w}$-closed in $\operatorname{Prim}(Q)$. Let $Q$ be in the closure of $V$. There is a net $\left(P_{\alpha}\right)_{\alpha}$ in $V$ converging to $Q$. By compactness there is a subnet $\left(P_{\beta}\right)_{\beta}$ converging to a point $P$ in $V$. But then $P_{\beta} \rightarrow\{P, Q\}$ and by (i) we conclude $Q=P \in V$.

(iii) Let $P \in U, Q \in \mathrm{Pl}(\mathcal{Q})$, and $\Phi(P)=\Phi(Q)$. Then $P=Q$. (First notice that this will finish the proof.) Let $Q_{0}$ be any primitive ideal containing $Q$. We have to prove $P=Q_{0}$, so without loss of generality let $Q$ be primitive. Assume $P \neq Q$. Then there is a compact neighbourhood $V$ of $P$ in $U$ such that $Q \notin V$, because $U$ is a locally compact Hausdorff space. Moreover there is a continuous function $f: U \rightarrow[0,1]$ such that $f(P)=1$ and $\operatorname{supp}(f) \subset V$. Let $F$ be the extension of $f$ to $\operatorname{Prim}(J)$, where $F$ is defined to be zero outside $U$. Let us prove that $F$ is in $\mathcal{C}_{0}(\operatorname{Prim}(J))$. Since the support of $F$ is contained in $\bar{V}^{\tau_{w}}$ which equals $V$ by (ii), we only have to check continuity. To this end let $R_{\alpha} \rightarrow R$ in Prim $(J)$. If $R \in U$, then $R_{\alpha} \in U$ for large $\alpha$, and so $F\left(R_{\alpha}\right)=$ $f\left(R_{\alpha}\right) \rightarrow f(R)=F(R)$. If $R \notin U$ then $R_{\alpha} \in \operatorname{Prim}(J) \backslash V$ for large $\alpha$ by (ii), and so again $F\left(R_{\alpha}\right) \rightarrow F(R)$. Thus indeed $F$ is continuous. By [10, Prop. 1] there is $a \in \operatorname{cen}(J) \subset \operatorname{cen}(Q)$ such that $\hat{a}=F$. Obviously $\hat{a}(P)=1 \neq 0=\hat{a}(Q)$, contradicting $\Phi(P)=\Phi(Q)$. This completes the proof.

COROllary 2.3. Let $Q$ be a separable liminal and unital $C^{*}$-algebra. Using the notation of the above proof, $\Phi: U \rightarrow \Phi(U)$ is a homeomorphism onto an open and dense subset of $T$.

Proof. By the preceding proof (especially part (iii)), $\Phi: U \rightarrow \Phi(U)$ is a continuous bijection. Let $t_{\alpha}=\Phi\left(P_{\alpha}\right) \rightarrow t=\Phi(P)$ in $T$. By the compactness of 
$\operatorname{Prim}(\mathbb{Q})$, any subnet of $\left(P_{\alpha}\right)_{\alpha}$ has a convergent subnet $P_{\beta} \rightarrow Q$ in $\operatorname{Prim}(\mathbb{Q})$. Then $\Phi(P)=t=\lim _{\beta} \Phi\left(P_{\beta}\right)=\Phi(Q)$, hence $P=Q$ by part (iii) of the above proof. This shows $P_{\alpha} \rightarrow P$; hence $\left.\Phi\right|_{U}$ is a homeomorphism.

Thus $\Phi(U)$ must be a locally compact subspace of $T$, and so it is an intersection of an open and closed subset of $T$. Since $\Phi(U)$ clearly is dense in $T$ (as $U$ is dense in $\operatorname{Prim}(Q)$ ), it must be open.

\section{Quasistates}

A quasistate on a $C^{*}$-algebra $Q$ is a map $f: Q \rightarrow \mathbf{C}$ such that

(i) if $B \subset Q$ is a commutative sub-C*-algebra, then $\left.f\right|_{B}$ is a positive linear functional;

(ii) $f(a+i b)=f(a)+i f(b)$ for all self-adjoint elements $a, b \in Q$;

(iii) $\sup \{f(a) \mid a \in \mathbb{Q}, a \geq 0,\|a\| \leq 1\}=1$.

The link to the original definition is given by [1].

It is an important and old problem to determine whether quasistates are linear. Gleason [18] has shown that this is indeed the case for $Q=M_{n}(\mathbf{C})$, $n \geq 3$, and wrong for $n=2$. This has been extended to von Neumann algebras without central summands of type $I_{2}$ by Christensen [8] and Yeadon [21].

General $C^{*}$-algebras are also treated in the literature. Aarnes [2] proved among other things that all continuous quasistates are linear if $Q$ is a liminal $C^{*}$-algebra with Hausdorff spectrum which does not possess any irreducible representations of dimension 2. Christensen [8] has shown that the continuity of the quasistates is automatic if $Q$ is a locally trivial field of elementary $\mathrm{C}^{*}$-algebras.

Also recall the following result of Kaplan [19], which is the starting point in [19] for treating $C^{*}$-algebras which are not von Neumann algebras: If $\mathbb{Q}$ is a separable unital $C^{*}$-algebra that possesses an ideal $B$ which is a locally trivial continuous field of elementary $C^{*}$-algebras such that $Q$ does not have any 2-dimensional irreducible representations, then all weakly subadditive quasistates on $\mathfrak{Q}$ are linear.

In order to generalize these results (partially), we will show as in [2] that in some situations an extremal continuous quasistate $f$ on $\mathbb{Q}$ induces a continuous quasistate $\tilde{f}$ on $Q / K$, where $K$ is a closed two-sided ideal. We will deduce linearity from this.

THEOREM 3.1. Let $\mathbb{Q}$ be a $C^{*}$-algebra possessing an essential separable quasicentral and liminal ideal. Let $f$ be a continuous quasistate on $\mathbb{Q}$ such that

$$
\operatorname{ker}\left(\left.f\right|_{\operatorname{cen}(Q)}\right)=\left\{a \in \operatorname{cen}(Q) \mid a\left(t_{0}\right)=0\right\}
$$

for some $t_{0} \in T_{\infty}$. Let $K:=\bigcap \Phi^{-1}\left(t_{0}\right)$. Then $\tilde{f}(a+K):=f(a)$ defines a continuous quasistate on $\mathbb{Q} / K$.

REMARK. Using the proof of [2, Lemma 5.2], it is easily seen that extremal continuous quasistates of $\mathfrak{Q}$ satisfy the assumption in the theorem. 
The space $\operatorname{Prim}(Q)$ is used in [2] in two ways. First, it serves as a space of ideals, where an ideal $P$ can be found such that the given extremal quasistate induces a quasistate on $Q / P$. Second, Prim $(Q)$ is used as a Hausdorff space, where the elements of the center of the multiplier algebra live as continuous functions. This multiple use of $\operatorname{Prim}(\mathfrak{Q})$ is not possible here. The space of ideals will be $\operatorname{MP}(\mathscr{Q})$, and the Hausdorff space will be $T$, the Gelfand space of the center. This causes some technical difficulties not present in [2], and we now show how to manage them.

Proof. If $Q$ has no unit, then adjoin one. The situation remains unchanged if we extend $f$ by $f(1)=1$ [2, par. 2]. So we assume that $Q$ is unital. The proof is broken up into several assertions.

Claim 1: Let $a \in \mathcal{Q}$ and $t \in T$. Then the map $I \mapsto\|a+I\|$ restricted to $\cap \Phi^{-1}(t)$ attains its maximum in a minimal primal ideal.

There is nothing to prove if $\psi: I \mapsto\|a+I\|$ is zero on $\Phi^{-1}(t)$. Otherwise let $\alpha>0$ be such that $\alpha<\sup \left\{\|a+I\| \mid I \in \Phi^{-1}(t)\right\}$. Since $\{I \mid\|a+I\| \geq \alpha\}$ is $\tau_{s^{-}}$ compact in $\operatorname{Id}(\mathscr{Q})$, hence in $\operatorname{Id}(\mathscr{Q}) \backslash\{\mathfrak{Q}\}$, and since $\Phi^{-1}(t)$ is $\tau_{w^{-}}$and hence $\tau_{s^{-}}$ closed in $\mathrm{Pl}(Q)$, we see that $\left\{I \in \Phi^{-1}(t) \mid\|a+I\| \geq \alpha\right\}$ is $\tau_{s}$-compact. But $\psi$ is $\tau_{s}$-continuous, and so the maximum will be attained in an ideal $I$. So the maximum is attained in any minimal primal ideal contained in $I$.

Claim 2: Let $a \in \mathbb{Q}$. Then there is a cross-section $\phi_{a}: T \rightarrow \operatorname{MP}(\mathbb{Q})$ of $\Phi$ such that $t \mapsto\left\|a+\phi_{a}(t)\right\|$ is upper semicontinuous and bounded by $\|a\|$.

By claim 1, there is a minimal primal ideal $\phi_{a}(t)$ such that the map $I \mapsto$ $\|a+I\|$ attains the maximum value on $\Phi^{-1}(t)$ at $\phi_{a}(t)$. It will be proved now that $\phi_{a}$ has the required properties. Let $t_{\alpha} \rightarrow t$ in $T$, and let us prove that $\lim \sup _{j}\left\|a+\phi_{a}\left(t_{j}\right)\right\| \leq\left\|a+\phi_{a}(t)\right\|$. Assuming the contrary, there is $\lambda \in \mathbf{R}$ such that

$$
\left\|a+\phi_{a}(t)\right\|<\lambda<\limsup _{j}\left\|a+\phi_{a}\left(t_{\alpha}\right)\right\|=: M .
$$

There is a subnet $\left(t_{\beta}\right)$ such that $\lambda<\left\|a+\phi_{a}\left(t_{\beta}\right)\right\| \rightarrow M$. Because $\{I \in \operatorname{Id}(\mathscr{Q}) \mid$ $\|a+I\| \geq \lambda\}$ is $\tau_{s}$-compact, there is a subnet $\left(t_{\gamma}\right)_{\gamma}$ of $\left(t_{\beta}\right)_{\beta}$ such that $\phi_{a}\left(t_{\gamma}\right) \rightarrow I$ in the strong topology and $\|a+I\| \geq \lambda$. Since

$$
I \in \operatorname{Pl}(\mathcal{Q}) \text { and } \Phi(I)=\lim \Phi\left(\phi_{a}\left(t_{\gamma}\right)\right)=t,
$$

we have $I \in \Phi^{-1}(t)$, and so $\|a+I\| \leq\left\|a+\phi_{a}(t)\right\|<\lambda$, which is a contradiction. So $\phi_{a}$ is upper semicontinuous. The boundedness is obvious.

Claim 3: $K=\bigcap \Phi^{-1}\left(t_{0}\right) \subset \operatorname{ker}(f)$.

Let $a \in K, a \geq 0$, and let $\epsilon>0$ be given. By claim 2 and since $\left\|a+\phi_{a}\left(t_{0}\right)\right\|=$ 0 , there exists $h \in \mathcal{C}(T)=\operatorname{cen}\left(Q_{1}\right)$ such that $h(t) \geq\left\|a+\phi_{a}(t)\right\|$ on $T$ and $h\left(t_{0}\right)<\epsilon$.

If $\pi$ is any irreducible representation of $Q$ then $\|\pi(a)\|=\|a+\operatorname{ker} \pi\| \leq$ $\left\|a+\phi_{a}(\Phi(\operatorname{ker} \pi))\right\| \leq\|h(\Phi(\operatorname{ker} \pi))\|=\pi(h)$, which is a scalar operator. So $\pi(h-a) \geq 0$ for all irreducible representations of $Q$, hence $a \leq h$, and therefore $-h\left(t_{0}\right) \leq a-h\left(t_{0}\right) \leq h-h\left(t_{0}\right)$. Now $f$ can be extended to a quasistate 
on $Q_{1}$. Then the restriction to the commutative $C^{*}$-algebra generated by $\{a, h, 1\}$ is linear, hence monotone, and so we get $-h\left(t_{0}\right) \leq f(a)-h\left(t_{0}\right) \leq$ $f(h)-h\left(t_{0}\right)=0$ by the assumption about $f$. Thus $0 \leq f(a) \leq \epsilon$, and this implies $a \in \operatorname{ker}(f)$. So $\operatorname{ker}(f)$ contains all positive elements in $K$. Since selfadjoint elements in $K$ can be written as the difference of two positive and commuting elements in $K$, we conclude that $\operatorname{ker}(f)$ contains all self-adjoint elements of $K$. Considering real and imaginary parts separately, we finally arrive at the claimed result.

As in [2], we cannot deduce directly that $f(a)=f(b)$ if $a+K=b+K$. This requires an additional argument.

Claim 4: Let $h \in \operatorname{cen}\left(\mathfrak{Q}_{1}\right)$ be self-adjoint, $h\left(t_{0}\right)=1, a \in \mathfrak{Q}$. Then $f(a)=$ $f(h a)$.

This can be proved as in [2, Lemma 6.1].

Claim 5: If $a, b \in Q$ and $a+K=b+K$, then $f(a)=f(b)$.

Let $\epsilon>0$ be given. Since $f$ is continuous, there is a $\delta>0$ such that $\|a-c\|<$ $\delta$ implies $|f(a)-f(c)|<\epsilon$. Let $\phi_{a-b}$ be the cross-section constructed in claim 2. The map $t \mapsto\left\|a-b+\phi_{a-b}(t)\right\|$ is upper semicontinuous and vanishes at $t_{0}$ since $a-b \in K \subset \phi_{a-b}\left(t_{0}\right)$. So there is an open neighbourhood $U$ of $t_{0}$ satisfying $\left\|a-b+\phi_{a-b}(t)\right\|<\delta$ on $U$. Let $C \subset U$ be a compact neighbourhood of $t_{0}$. Choose continuous functions $g$ and $h$ from $T$ to $[0,1]$ such that $h\left(t_{0}\right)=1$, $\operatorname{supp}(h) \subset C$, and $\left.g\right|_{C}=1, \operatorname{supp}(g) \subset U$. Define $c:=g b+(1-g) a \in \mathcal{Q}$. Then

$$
\left\|a-c+\phi_{a-b}(t)\right\|=g(t)\left\|a-b+\phi_{a-b}(t)\right\|<\delta,
$$

and so $\sup _{t \in T}\left\|a-c+\phi_{a-b}(t)\right\| \leq \delta$. By Theorem 2.2 and using the $\tau_{s}$-continuity of the map $I \mapsto\|a-c+I\|$, we conclude that $\|a-c\| \leq \delta$ and hence $|f(a)-f(c)|<\epsilon$.

Now we may go on as in [2, Lemma 6.3]. We have $h c=h g b=h b$, and by claim 4 this means $f(c)=f(b)$. Since $\epsilon$ was arbitrary, we have $f(a)=f(b)$.

This clearly finishes the proof of the theorem.

This section concludes with two applications.

TheOREm 3.2. Let $Q$ be a liminal separable $C^{*}$-algebra such that

(1) $Q$ does not possess any irreducible representations of dimension 2, and

(2) if $M \subset \operatorname{Prim}(Q)$ is infinite then there exists an $a \in \operatorname{cen}(Q)$ such that $\hat{a}$ is nonconstant on $M$.

Then all continuous quasistates are linear.

Proof. Let $Z:=\{P \in \operatorname{Prim}(Q) \mid \hat{a}(P)=0$ for all $a \in \operatorname{cen}(Q)\}$. By assumption (2) $Z$ is finite, hence $Z_{0}:=\operatorname{int}(Z)$ is a finite set of one-element components of $\operatorname{Prim}(\mathfrak{Q})$. So $Q$ decomposes as $Q=\oplus_{P \in Z_{0}} Q / P \oplus \cap Z_{0}$. Since $\mathfrak{Q}$ is liminal, by (1) we have $Q / P \cong M_{n}(\mathbf{C})$, where the positive integer $n$ is different from 2 and where $M_{\infty}$ stands for the compact operators. So by Gleason's 
result [18], any continuous quasistate is linear on $\oplus_{P \in Z_{0}} Q / P$. Hence we may assume that $Z_{0}$ is empty.

Let $J$ be the ideal $\cap Z$. Since $Z$ has no interior points, it is an essential ideal, and is of course also separable and liminal. By definition of $J$ we have $\operatorname{cen}(J)=\operatorname{cen}(Q)$ (cf. [11, Lemme 6]), and so $J$ is quasicentral.

As already observed in [2], it is enough to prove that any extremal quasistate $f$ is linear. But then $Q$ and $f$ satisfy all the assumptions of Theorem 3.1, and $f$ gives rise to a continuous quasistate $\tilde{f}$ on $Q / \Phi^{-1}\left(t_{0}\right)$ for some $t_{0} \in T_{\infty}$. But $\Phi^{-1}\left(t_{0}\right)$ is finite by assumption (2), and by $[12,4.2 .5]$ we conclude that this quotient $C^{*}$-algebra is isomorphic to a finite direct sum of matrix algebras. So again by Gleason's result [18] we conclude that $\tilde{f}$ is linear, and so must $f$ be.

One of the starting points for treating general $C^{*}$-algebras is [19, Prop. 3.4], cited previously. Since weakly subadditive quasistates on unital $\mathrm{C}^{*}$-algebras are continuous [19, Prop. 2.2], the following theorem generalizes this proposition in the case where the fibres of $B$ in [19, Prop. 3.4] are finite-dimensional.

Theorem 3.3. Let $Q$ be a separable $C^{*}$-algebra possessing a liminal quasicentral ideal $J$ such that:

(1) Q does not possess any irreducible representation of dimension 2;

(2) If $M \subset \operatorname{Prim}(J)$ is infinite then there exists an $a \in \operatorname{cen}(J)$ such that $\hat{a}$ is nonconstant on $M$; and

(3) $Q / J$ is finite-dimensional.

Then all continuous quasistates of $\mathbb{Q}$ are linear.

Proof. We have $\operatorname{Prim}(Q)=\operatorname{Prim}(J) \cup \operatorname{Prim}(Q / J)$, where $\operatorname{Prim}(Q / J)$ is finite by assumption (3). Using the same arguments as in the proof of Theorem 3.2 , we may assume that int $(\operatorname{Prim}(\mathscr{Q} / J))$ is empty-that is, $J$ is essential.

If $f$ is a pure quasistate on $Q$, the conditions of Theorem 3.1 are fulfilled and hence $f$ gives rise to a continuous quasistate $\tilde{f}$ on $Q / \Phi^{-1}\left(t_{0}\right)$ for some $t_{0} \in T_{\infty}$. By (2) and (3) $\Phi^{-1}\left(t_{0}\right)$ is finite, and since $J$ is quasicentral all irreducible representations are finite-dimensional. So the above quotient $\mathrm{C}^{*}$ algebra also is finite-dimensional and all its irreducible representations have dimension different from 2 by assumption (1). Again we may conclude that $\tilde{f}$, and hence $f$, is linear.

\section{Quasitraces and Dimension Functions}

From the preceding section we immediately have this theorem.

THEOREM 4.1. Let $Q$ be a liminal separable $C^{*}$-algebra such that

(*) if $M \subset \operatorname{Prim}(\mathfrak{Q})$ is infinite then there exists an $a \in \operatorname{cen}(\mathfrak{Q})$ such that $\hat{a}$ is nonconstant on $M$.

Then all continuous quasitraces are linear. 
To see this, use the same arguments for quasitraces instead of quasistates. The only fact that deserves an extra argument is that extremal quasitraces satisfy the assumptions of Theorem 3.1, but the arguments in [2, Lemma 5.2] will work. Finally, use the fact that continuous quasistates on $M_{2}(\mathbf{C})$ are linear (there are not so many).

There is an interesting connection between lower semicontinuous dimension functions and continuous quasitraces. This can be found in [9] or [6]. If $D$ is a lower semicontinuous dimension function then the following construction gives a continuous quasitrace.

Let $a \in Q$ be self-adjoint. If $U \subset \mathbf{C}$ is open, choose a continuous function $g: \mathbf{C} \rightarrow \mathbf{R}_{0}^{+}$such that $U=\{z \in \mathbf{C} \mid g(z)>0\}$. Define $\mu_{a}(U)=D(g(a))$. This can be extended to a Borel probability measure on $\mathbf{C}$ which is concentrated on the spectrum of $a$. Then let $\tau(a):=\int_{\mathbf{C}} \lambda d \mu_{a}(\lambda)$. This defines a continuous quasitrace on $Q$.

Conversely, let a continuous quasitrace $\tau$ on $Q$ be given. Define $f_{\epsilon}: \mathbf{R} \rightarrow \mathbf{R}$ by $f_{\epsilon}(t)=0$ for $t \leq \epsilon / 2, f_{\epsilon}(t)=2 t / \epsilon-1$ for $\epsilon / 2 \leq t \leq \epsilon$, and $f_{\epsilon}(t)=1$ for $t \geq \epsilon$. Then $D(x):=\lim _{\epsilon \rightarrow 0} \tau\left(f_{\epsilon}(|x|)\right)$ defines a lower semicontinuous rank function on $Q$. If $\tau$ actually is a trace then we have a dimension function.

The coincidence of traces and quasitraces is a useful piece of information about a $C^{*}$-algebra.

The lower semicontinuous dimension functions on a commutative $\mathrm{C}^{*}$ algebra $C_{0}(T)$ are described in [6, Prop. I.2.1]. Let $\mu$ be a measure on the $\sigma$-algebra generated by the $\sigma$-compact open sets in $T$. For $x \in C_{0}(T)$, define $D_{\mu}(x):=\mu(\{t \in T \mid f(t) \neq 0\})$. Then $D_{\mu}$ is a lower semicontinuous dimension function, and all other lower semicontinuous dimension functions arise in this way.

The following theorem gives a similar description for a class of noncommutative $C^{*}$-algebras. Let $S(Q)$ denote the set of positive linear functionals on $Q$ with norm not exceeding 1 .

Theorem 4.2. Let $\mathfrak{Q}$ be a separable liminal and quasicentral $C^{*}$-algebra such that all quasistates are linear.

(1) Let $D$ be a lower semicontinuous dimension function on $Q$. Let $U \subset$ $\operatorname{Prim}(Q)$ be open and let $I$ be the corresponding ideal of $Q$. There exists an $x \in I^{+}$such that $\left\{\phi \in S(Q) \mid \operatorname{ker}\left(\pi_{\phi}\right) \not \supset I\right\}=\{\phi \in S(Q) \mid \phi(x)>0\}$. Define $\mu(U):=D(x)$. This is well-defined and can be extended to a Borel probability measure $\mu_{D}$ on $\operatorname{Prim}(\mathfrak{Q})$.

(2) Let $\mu$ be a Borel probability measure on Prim( $(\mathfrak{Q})$. Then

$$
D_{\mu}(x):=\int_{\operatorname{Prim}(\alpha)} \frac{\operatorname{dim}(\operatorname{im} \pi(x))}{\operatorname{dim} \pi} d \mu(\pi)
$$

(3) The maps $D \mapsto \mu_{D}$ and $\mu \mapsto D_{\mu}$ are inverses of each other.

The proof is broken into a series of lemmata. First notice that the assumptions on $Q$ imply that all irreducible representations are finite-dimensional. 
LEMMA 4.3. If I is a closed two-sided ideal of $Q$ then there is an element $x \in I^{+}$such that $\left\{\phi \in S(Q) \mid \operatorname{ker}\left(\pi_{\phi}\right) \not \supset I\right\}=\{\phi \in S(\mathbb{Q}) \mid \phi(x)>0\}$. If $D$ is a lower semicontinuous dimension function then $D(x)$ is the same number for all such $x$.

Proof. Let $\left(x_{n}\right)_{n}$ be a countable approximate unit for $I$, and let $\left(a_{n}\right)_{n}$ be a countable dense set in the unit ball of $Q$. Then $I^{+}$contains

$$
x:=\sum_{n, m} \frac{1}{2^{n+m}} a_{n}^{*} x_{m} a_{n} .
$$

If $\phi \in S(Q)$ and $\phi(x)>0$ then clearly $\pi_{\phi}(x) \neq 0$, hence $\operatorname{ker}\left(\pi_{\phi}\right) \not \supset I$. Conversely, let $\operatorname{ker}\left(\pi_{\phi}\right) \not \supset I$. Define $\tilde{x}:=\Sigma_{m}\left(1 / 2^{m}\right) x_{m}$. If we had $\tilde{x} \in \operatorname{ker}\left(\pi_{\phi}\right)$, it would contain $x_{1}, x_{2}, \ldots$, and this would imply $I \subset \operatorname{ker}\left(\pi_{\phi}\right)$ which is not the case. So there exists a $\xi \in H_{\pi},\|\xi\|=1$, such that $\left\langle\pi_{\phi}(\tilde{x}) \xi \mid \xi\right\rangle>0$. If $\xi_{\phi}$ is the cyclic vector belonging to $\phi$ then there must be an $n \in \mathbf{N}$ such that

$$
\left\langle\pi_{\phi}(\tilde{x}) \pi_{\phi}\left(a_{n}\right) \xi_{\phi} \mid \pi_{\phi}\left(a_{n}\right) \xi_{\phi}\right\rangle>0,
$$

hence $\phi\left(a_{n}^{*} \tilde{x} a_{n}\right)>0$, hence $\phi(x)>0$. So the existence of such an $x$ is proved.

Now let $y \in I^{+}$be another such element. We have to prove $D(x)=D(y)$. Let $Q \subset L(H)$ be given in its universal representation [20, chap. 3.7].

Let us first prove that $\operatorname{im}(x)$ and $\operatorname{im}(y)$ have the same closure. To this end let $\xi \in H$ and $\eta \in \operatorname{ker} y$. Then $|\langle x \xi \mid \eta\rangle|^{2} \leq\langle x \xi \mid \xi\rangle\langle x \eta \mid \eta\rangle=0$, since $\langle y \eta \mid \eta\rangle=0$ implies $\langle x \eta \mid \eta\rangle=0$ by the properties of $x$ and $y$. So we have $\operatorname{im}(x) \subset \overline{\operatorname{im}(y)}$, and by symmetry $\overline{\operatorname{im}(x)}=\overline{\operatorname{im}(y)}$.

Since $y(y+1 / n)^{-1}$ increases to the projection $p$ onto $\overline{\operatorname{im}(y)}$ in the strong operator topology, we have $\phi\left(x\left(1-y(y+1 / n)^{-1}\right) x\right) \searrow \phi(x(1-p) x)=0$ for all $\phi \in S(\mathbb{Q})$. By Dini's theorem we conclude that $\left\|x\left(1-y(y+1 / n)^{-1}\right) x\right\| \rightarrow 0$, hence $x \leqslant x^{2} \leqslant y$ (notation as in [6] or [9]). This implies $D(x) \leq D(y)$, and a symmetry argument establishes the lemma.

So for an open subset $U$ of $\operatorname{Prim}(Q)$ we can define $\mu_{D}(U)$ as stated in the theorem. If it were possible to extend this function to a Borel measure on $\operatorname{Prim}(\mathfrak{Q})$, this extension would clearly be unique. Since Prim $(\mathfrak{Q})$ may be nonHausdorff, the existence seems not to be clear.

Let $T(Q)$ be the compact convex set of traces on $Q$ with norm not exceeding 1. As explained in [15, chap. 2] there is a map $\Theta: \operatorname{ex} T(Q) \backslash\{0\} \rightarrow \operatorname{Prim}(\mathscr{Q})$ given by $\Theta(\tau)=\operatorname{ker} \pi_{\tau}$, where $\pi_{\tau}$ is the GNS-representation defined by $\tau$. It is easy to see that $\Theta$ is continuous when $\operatorname{ex} T(Q)$ is equipped with the relative weak*-topology (we don't need the facial topology here).

Now let $D$ be a lower semicontinuous dimension function. $D$ determines a quasitrace $\tau_{0}$ which by assumption is an element of $T(\mathbb{Q})$. So by Choquet's theorem there is a Borel probability measure $\mu_{0}$ concentrated on ex $T(Q)$ such that $\tau_{0}(x)=\int_{\mathrm{ex} T(Q)} \tau(x) d \mu_{0}(\tau)$ for all $x \in \mathcal{Q}$. Let $\mu_{1}:=\mu_{0}{ }^{\circ} \Theta^{-1}$, which is a Borel probability measure on $\operatorname{Prim}(\mathscr{Q})$.

Lemma 4.4. If $U \subset \operatorname{Prim}(\mathfrak{Q})$ is open then $\mu_{1}(U)=\mu_{D}(U)$. 
Proof.

$$
\begin{aligned}
\mu_{1}(U) & =\mu_{0}\left(\left\{\tau \in \operatorname{ex} T(Q) \mid \operatorname{ker} \pi_{\tau} \not \supset I\right\}\right) \\
& =\mu_{0}(\{\tau \in \operatorname{ex} T(Q) \mid \tau(x)>0\}) \quad(\text { where } x \text { is chosen by Lemma 4.3) } \\
& =\int_{\operatorname{ex} T(Q)} \chi_{\{\sigma \mid \hat{x}(\sigma)>0\}}(\tau) d \mu(\tau),
\end{aligned}
$$

where $\hat{x}(\sigma):=\sigma(x)$; that is, $x$ operates as a continuous function on $T(\mathcal{Q})$. But

$$
\mu_{D}(U)=D(x)=\lim _{\epsilon \rightarrow 0} \tau_{0}\left(f_{\epsilon}(x)\right)=\lim _{\epsilon \rightarrow 0} \int_{\operatorname{ex} T(\alpha)} \widehat{f_{\epsilon}(x)}(\tau) d \mu_{0}(\tau) .
$$

So by the monotone convergence theorem we need only prove that $\widehat{f_{\epsilon}(x)}$ increases to $\chi_{\{\sigma \mid \hat{x}(\sigma)>0\}}$ as $\epsilon$ decreases to zero.

We have $\widehat{f_{\epsilon}(x)}(\tau)=\left\langle f_{\epsilon}\left(\pi_{\tau}(x)\right) \xi_{\tau} \mid \xi_{\tau}\right\rangle>\left\langle p \xi_{\tau} \mid \xi_{\tau}\right\rangle$, where $p$ is the projection onto $\operatorname{im} \pi_{\tau}(x)$. Let us show that $p$ is zero or one.

To this end let $y \in I$ be a self-adjoint element, $\eta \in\left(\operatorname{im} \pi_{\tau}(x)\right)^{\perp}$. Let $\left(x_{n}\right)_{n}$, $\tilde{x}$, and $a_{n}$ be used (as in the proof of Lemma 4.3) for the construction of $x$. Without loss of generality we may assume that $a_{1}$ is a scalar multiple of $\tilde{x}^{1 / 2}$. Then a scalar multiple of $x^{1 / 2}$ dominates $\tilde{x}$. For $\epsilon>0$ we have

$$
y^{2}-\epsilon \leq x_{m} y^{2} x_{m} \leq\left\|y^{2}\right\| x_{m}
$$

for large $m$. By the construction of $x$ we have $x_{m} \leq \lambda x^{1 / 2}$ and so $y^{2} \leq \lambda x^{1 / 2}+\epsilon$ for some large $\lambda$. Then $\left\|\pi_{\tau}(y) \eta\right\|^{2} \leq\left\langle\lambda \pi_{\tau}\left(x^{1 / 2}\right) \eta \mid \eta\right\rangle+\epsilon\langle\eta \mid \eta\rangle=\epsilon\|\eta\|^{2}$. So we have proved that $\left(\operatorname{im} \pi_{\tau}(x)\right)^{\perp} \subset \operatorname{ker} \pi_{\tau}(y)$ and hence $\overline{\operatorname{im} \pi_{\tau}(y)} \subset \overline{\operatorname{im} \pi_{\tau}(x)}$ for all self-adjoint elements $y \in I$, and this implies

$$
\overline{\operatorname{im} \pi_{\tau}(x)}=\overline{\operatorname{span} \cup\left\{\operatorname{im} \pi_{\tau}(y) \mid y \in I\right\}} .
$$

The set on the right clearly is $\pi_{\tau}(\mathfrak{Q})$-invariant, so $p$ is in the center of $\pi_{\tau}(\mathfrak{Q})$. Thus $p$ must be one or zero as $\tau$ is an extremal trace; that is, $\pi_{\tau}$ is a factor representation.

Now if $\hat{x}(\tau)=0$ then $\pi_{\tau}(x)=0$, hence $p=0$. If $\hat{x}(\tau)>0$ then $p=0$ is impossible, hence $p=1$ and hence $\widehat{f_{\epsilon}(x)}(\tau) \nearrow\left\langle p \xi_{\tau} \mid \xi_{\tau}\right\rangle=1$.

This also finishes the proof of part (1) of the theorem. We now will turn to part (2).

\section{Lemma 4.5. The map}

$$
\rho: \operatorname{Prim}(Q) \rightarrow \mathbf{R}, \quad \rho(\pi)=\frac{\operatorname{dim}(\operatorname{im} \pi(|x|))}{\operatorname{dim} \pi},
$$

is Borel measurable for each $x \in \mathcal{Q}$. If $\mu$ is a Borel measure on Prim $(\mathcal{Q})$ then $D_{\mu}$ is a lower semicontinuous dimension function on $Q$.

Proof. By $[12,3.6 .3]$ the map $\pi \mapsto \operatorname{dim} \pi$ is measurable; especially

$$
\operatorname{Prim}(\mathscr{Q})_{n}:=\{\pi \mid \operatorname{dim} \pi=n\}
$$


is a measurable set. By the proof of $[12,4.6 .2]$ there is a measurable crosssection $s: \operatorname{Prim}(\mathscr{Q})_{n} \rightarrow \operatorname{Irr}_{n}(\mathscr{Q})$, where the latter set stands for the irreducible representations of $Q$ onto $L\left(H_{n}\right)$ for a fixed $n$-dimensional Hilbert space $H_{n}$, and where this set is endowed with the relative weak operator topology. The map $\pi \mapsto \pi(|x|)$ is continuous, and so $\pi \mapsto \operatorname{dim} \pi(|x|)$ is measurable on $\operatorname{Irr}_{n}(Q)$. But then $[\pi] \mapsto \operatorname{dim}(\operatorname{im} \pi(|x|))=\operatorname{dim}(\operatorname{im} s([\pi])(|x|))$ is measurable and so is the quotient $\rho$ on $\operatorname{Prim}(\mathfrak{Q})_{n}$.

This proves the stated measurability; simple computations show that $D_{\mu}$ is a lower semicontinuous subadditive rank function and hence a dimension function [6, II.3.1].

LEMMA 4.6. $\quad D_{\mu_{D}}=D$.

Proof. (1) If $\tau \in \operatorname{ex} T(\mathfrak{Q}) \backslash\{0\}$ then $D_{\tau}(x)=\operatorname{dim}\left(\operatorname{im} \pi_{\tau}(|x|) / \operatorname{dim} \pi_{\tau}\right.$. Because $\tau$ is extremal, the corresponding GNS-representation has the image $L\left(H_{n}\right)$ and hence $T \circ \pi_{\tau}=\tau$, where $T$ is the unique normalized trace on $H_{n}$. Thus $D_{\tau}(x)=\lim _{\epsilon \rightarrow 0} T\left(f_{\epsilon}\left(\left|\pi_{\tau}(x)\right|\right)\right)$ gives the desired formula.

(2) $D$ induces a (quasi)trace $\tau_{0}$ on $Q$, so

$$
\tau_{0}(x)=\int_{\operatorname{ex} T(Q) \backslash\{0\}} \tau(x) d \mu_{0}(\tau) \text { for all } x \in Q,
$$

where $\mu_{0}$ is some probability measure on ex $T(Q)$. By Lemma 4.4 we have $\mu_{D}=\mu_{0} \circ \Theta^{-1}$; hence

$$
\begin{aligned}
D_{\mu_{D}}(x) & =\int_{\operatorname{Prim}(\Theta)} \frac{\operatorname{dim}(\operatorname{im} \pi(|x|))}{\operatorname{dim} \pi} d \mu_{D}(\pi)=\int_{\operatorname{ex} T(())} D_{\tau}(x) d \mu_{0}(\tau) \\
& =\lim _{\epsilon \rightarrow 0} \int_{\operatorname{ex} T(Q)} \tau\left(f_{\epsilon}(|x|)\right) d \mu_{0}(\tau)=\lim _{\epsilon \rightarrow 0} \tau_{0}\left(f_{\epsilon}(|x|)\right)=D(x) .
\end{aligned}
$$

LEMMA 4.7. $\mu_{D_{\mu}}=\mu$.

Proof. (1) Let $U \subset \operatorname{Prim}(Q)$ be open, say $U=\{P \mid P \not \supset I\}$ for some ideal $I$. Let $x \in I^{+}$be chosen as in Lemma 4.3. Then $\operatorname{dim}(\operatorname{im} \pi(x))=\operatorname{dim} \pi$ if and only if $\operatorname{ker} \pi \in U$, and also $\operatorname{dim}(\operatorname{im} \pi(x))=0$ if and only if $\operatorname{ker} \pi \notin U$.

To see this, let $\operatorname{dim}(\operatorname{im} \pi(x))<\operatorname{dim} \pi$. Then there is a unit vector $\xi \in H_{\pi}$ such that $\phi(x):=\langle\pi(x) \xi \mid \xi\rangle=0$. By the properties of $x$ we conclude that $\operatorname{ker} \pi=\operatorname{ker} \pi_{\phi} \supset I$ and hence $\operatorname{ker} \pi \notin U$.

If $\operatorname{dim}(\operatorname{im} \pi(x))=\operatorname{dim} \pi$ then $\pi(x)$ is invertible. So for any unital vector $\xi \in H_{\pi}$ we have $\phi(x):=\langle\pi(x) \xi \mid \xi\rangle>0$, and again by Lemma 4.3 we conclude that $\operatorname{ker} \pi \in U$. This proves claim (1).

(2) For $U \subset \operatorname{Prim}(Q)$ open, let $x$ be as in (1). Then by (1) we have

$$
\mu_{D_{\mu}}(U)=D_{\mu}(x)=\int_{\operatorname{Prim}(Q)} \frac{\operatorname{dim}(\operatorname{im} \pi(x))}{\operatorname{dim} \pi} d \mu(\pi)=\mu(U) .
$$

This finishes the proof of the theorem. 
EXAMPLE. Let

$$
\mathfrak{Q}:=\left\{x \in \mathcal{C}\left([0,1], M_{2}\right) \mid x(1 / n)=\operatorname{diag}\left(\lambda_{n}(x), \lambda_{n+1}(x)\right) \text { for all } n \in \mathbf{N}\right\} .
$$

It is easily seen that the irreducible representations of this separable liminal and unital $\mathrm{C}^{*}$-algebra are (up to unitary equivalence)

$\pi_{t}$, where $\pi_{t}(x):=x(t)$ for $\left.\left.t \in\right] 0,1\right] \backslash\{1 / n \mid n \in \mathbf{N}\}$;

$\lambda_{n}$ for $n \in \mathbf{N}$;

$\lambda_{0}$, where $\lambda_{0}(x):=x(0)_{1,1}=x(0)_{2,2}$.

If $t$ approaches $1 / n$ then $\pi_{t} \rightarrow\left\{\lambda_{n}, \lambda_{n+1}\right\}$, hence $\hat{x}\left(\lambda_{n}\right)=\hat{x}\left(\lambda_{n+1}\right)$ for all

$$
x \in \operatorname{cen}(Q)=\{x \in Q \mid x(t) \in \mathbf{C} 1 \text { and } x(1 / n)=x(0) \text { for all } n\} .
$$

So $M:=\left\{\lambda_{n} \mid n \in \mathbf{N} \cup\{0\}\right\}$ is an infinite set of Prim(Q) such that all central elements are constant on $M$.

It is easily seen that $Q$ is the inductive limit of the $C^{*}$-algebras

$$
\mathfrak{Q}_{n}:=\left\{x \in \mathbb{Q}|x|_{[0,1 / n]} \text { is constant }\right\} .
$$

Since all continuous quasitraces on $\mathfrak{Q}_{n}$ are linear by Theorem 4.1, we may conclude that all continuous quasitraces on $Q$ are linear. So by Theorem 4.2 we may describe all lower semicontinuous dimension functions for $Q$ by Borel measures on Prim( $(Q)$.

Considering this example, it would be interesting to know which $\mathrm{C}^{*}$ algebras may occur as an inductive limit of $C^{*}$-algebras satisfying the hypothesis of Theorem 4.1.

\section{The Minimal Primal Ideal Space as a Polish Space}

By [17], $\operatorname{Id}(Q)$ is a continuous lattice with respect to inclusion and $\tau_{s}$ is nothing but the Lawson topology, which is the common refinement of the Scott topology and the lower topology (see [17] for definitions). Let $L$ be a continuous lattice and $X \subset L$ a Lawson closed subset $\neq \emptyset$. Let us denote by $\min (X)$ (resp. $\max (X))$ the set of all minimal (resp. maximal) elements of $X$. By [17, III.1.10] and Zorn's lemma, these sets are nonempty. Another property, which will be useful in the sequel, is stated in the following theorem.

THEOREM 5.1. Let L be a continuous lattice such that the Lawson topology is second-countable, and let $X \subset L$ be a nonempty Lawson-closed set. Then $\min (X)$ and $\max (X)$ are Polish spaces in their respective topologies.

Proof. Since $L$ is a compact Hausdorff space [17, III.1.10] and secondcountable by assumption, it must be Polish. So we only have to prove that $\min (X)$ and $\max (X)$ are $G_{\delta}$-sets $[20,4.2 .2]$.

The sets $G_{1}:=\left\{(x, y) \in L^{2} \mid x \leq y\right\}$ and $G_{2}:=\left\{(x, y) \in L^{2} \mid x \geq y\right\}$ are closed in $L^{2}$. ( $L$ is meet-continuous by [17, I.2.2], $\sigma(L)$ is continuous by [17, II.1.14]; then use [17, III.2.4].) So $G:=X^{2} \cap G_{2} \backslash G_{1}$ is an $F_{\sigma}$-set and therefore $\sigma$-compact by the compactness of $L^{2}$. Hence $p_{1}(G)$ is $\sigma$-compact and therefore is an $F_{\sigma}$-set, where $p_{1}: L^{2} \rightarrow L$ denotes the projection onto the first coordinate. 
But $x \in X \backslash \min (X) \Leftrightarrow x \in X$, and there is a $y \in X$ such that $y \leq x$ and $x \notin y \Leftrightarrow$ there is a $y \in L$ such that $(x, y) \in X^{2} \cap G_{2} \backslash G_{1} \Leftrightarrow x \in p_{1}(G)$. Hence $\min (X)=X \cap\left(L \backslash p_{1}(G)\right)$ is the intersection of two $G_{\delta}$ 's and so is itself a $G_{\delta}$. The result for $\max (X)$ follows by exchanging $G_{1}$ and $G_{2}$.

Corollary 5.2. Let $\mathfrak{Q}$ be a separable $C^{*}$-algebra. Then $\mathrm{MP}(\mathfrak{Q})$ is a Polish space.

Proof. Observe that $\operatorname{Primal}(\mathscr{Q})$ is a $\tau_{s}$-closed subset of $\operatorname{Id}(\mathscr{Q})$ (which is second-countable by separability), and that $M P(Q)=\min (\operatorname{Primal}(\mathscr{Q}))$.

Corollary 5.3. Let $Q$ be a separable unital $C^{*}$-algebra. Then the space of maximal ideals is a Polish space with respect to $\tau_{s}$.

Proof. By [3, 4.1], $\operatorname{Id}(Q) \backslash\{Q\}$ is $\tau_{s}$-closed and hence Lawson-closed. So the theorem is applicable.

Let $\Phi: \mathrm{MP}(\mathscr{Q}) \rightarrow T_{\infty}$ be the canonical map.

THEOREM 5.4. There is a Borel measurable map $\psi: T \rightarrow \mathrm{MP}(\mathbb{Q})$ such that $\Phi \circ \psi=\mathrm{id}_{T}$.

Proof. By $[20,4.2 .12]$ we must prove that $\Phi^{-1}(s)$ is closed for all $s \in Z$ and that $\phi(G) \subset T$ is a Borel set for all open sets $G \subset \mathrm{MP}(\mathscr{Q})$. The first part trivially holds by continuity. So let $G \subset \mathrm{MP}(Q)$ be nonempty and open, and let us prove that $\Phi(G)$ is an $F_{\sigma}$-set, hence a Borel set.

There is a $\tau_{w}$-open set $U \subset \operatorname{Pl}(\mathfrak{Q})$ such that $G=U \cap \mathrm{MP}(\mathfrak{Q})$. Consider $P \in U$. Let $I$ be a minimal primal ideal contained in $P$. Then $I \in U$ (since $U$ is open) and therefore $\Phi(P)=\Phi(I)$, where $I \in U \cap \mathrm{MP}(Q)=G$. So we have proved one inclusion of $\Phi(U)=\Phi(G)$; the other is trivial. It remains to prove that $\Phi(U)$ is $F_{\sigma}$.

$U$ is also $\tau_{s}$-open, and so is a countable union of $\tau_{s}$-compact sets $K_{1}$, $K_{2}, \ldots$. But the $K_{n}$ are also $\tau_{w}$-compact (not necessarily Hausdorff), and so $\Phi(U)=\bigcup \Phi\left(K_{n}\right)$ is a countable union of compact and hence closed sets, since $T$ is Hausdorff.

For any $t \in T$, let $\mathcal{Q}(t):=\mathbb{Q} / \psi(t)$ for a fixed Borel cross-section $\psi: T \rightarrow$ $\operatorname{MP}(\mathcal{Q})$. So any $x \in \mathfrak{Q}$ defines a field $\tilde{x}$ of operators, where $\tilde{x}(t)=x+\psi(t) \in$ $Q / \psi(t)$. Let $\Psi$ be the map $x \rightarrow \tilde{x}$ into the $C^{*}$-algebra $\mathbb{B}$ of bounded operator fields on $T$ with fibres $Q(t)$, which is obviously a *-homomorphism.

From now on $Q$ must be unital. Then a complex-valued and bounded function on $T$ may be considered as an element in $B$; in particular, the ring of continuous functions $\mathcal{C}(T)$ is contained in $B$.

We may consider $\tilde{x}=\hat{x} \circ \psi$ as a complex-valued function on $T$. Since $\psi$ in general is not continuous (see the example below), the first part of the next theorem is surprising.

THEOREM 5.5. Using our previous notation, for a separable unital $C^{*}$ algebra $\propto$ we have: 
(i) $\Psi(\operatorname{cen}(\mathbb{Q}))=\mathfrak{C}(T)$.

(ii) $\|\tilde{x}(\cdot)\|$ is a Borel measurable function on $T$ for all $x \in \mathbb{Q}$.

(iii) If $f: T \rightarrow \cup Q(t)$ is an operator field such that for all $t_{0} \in T$ and $\epsilon>0$ there is an open neighbourhood $U$ of $t_{0}$ and an element $x \in \mathcal{Q}$ such that $\|f(t)-\tilde{x}(t)\|<\epsilon$ for all $t \in U$, then there is an element $y \in \mathcal{Q}$ such that $f=\tilde{y}$.

(iv) for all $t \in T$ and $a \in \mathcal{Q}(t)$ there is an $x \in \mathcal{Q}$ such that $\tilde{x}(t)=a$.

Proof. (i) Let $x \in \operatorname{cen}(\mathfrak{Q})$. To show that $\tilde{x}$ is continuous let $t_{\alpha} \rightarrow t$ in $T$. Let $Q_{\alpha}:=\psi\left(t_{\alpha}\right)$. By compactness [3, Prop. 4.1], any subnet of $\left(Q_{\alpha}\right)$ has a convergent subnet, say $Q_{\beta} \rightarrow I$ in $\operatorname{Pl}(\mathcal{Q})$. Then $\tilde{x}\left(t_{\beta}\right)=\hat{x}\left(Q_{\beta}\right) \rightarrow \hat{x}(I)$, and the continuity of $\tilde{x}$ will follow if we show that $\hat{x}(I)=\tilde{x}(t)$. But we have $t_{\beta}=$ $\Phi\left(Q_{\beta}\right) \rightarrow \Phi(I)$ and $t_{\beta} \rightarrow t$; that is, $\Phi(I)=t$. So $I$ and $\psi(t)$ are mapped to the same element by $\Phi$, and we have $\tilde{x}(t)=\hat{x}(\psi(t))=\hat{x}(I)$.

Conversely, let $f \in \mathrm{C}(T)$. Then $f \circ \Phi$ is a bounded and continuous function on Prim( $(\mathfrak{Q})$, and so by the Dauns-Hofmann theorem there is an $x \in \operatorname{cen}(\mathfrak{Q})$ such that if $f \circ \Phi=\hat{x}$ then $f=\tilde{x}$ is in the image of $\Psi$.

(ii) Clearly $\|\tilde{x}(\cdot)\|=\|\cdot\| \circ \hat{x} \circ \psi$. Since $\|\cdot\| \circ \hat{x}$ is continuous $[3,4.2]$, the claim follows from the Borel measurability of $\psi$.

(iii) The usual trick of using a partition of unity will do: Let $f$ and $\epsilon>0$ be given as in part (iii) of the theorem. By compactness of $T$, there are open sets $U_{1}, \ldots, U_{n}$ and elements $x_{1}, \ldots, x_{n} \in Q$ such that $T=\bigcup U_{j}$ and

$$
\sup _{t \in U_{i}}\left\|f(t)-\tilde{x}_{j}(t)\right\| \leq \epsilon .
$$

Let $\left(g_{j}\right)_{j}$ be a partition of unity subordinated to the cover $\left(U_{j}\right)_{j}$. Then the usual estimates show that $\left\|f(t)-\sum_{j=1}^{n} g_{j}(t) \tilde{x}_{j}(t)\right\| \leq \epsilon$ for all $t$. By (i) we know $g_{j}=\tilde{a}_{j}$ for some $a_{j} \in \operatorname{cen}(Q)$. So we have proved that

$$
\sup _{t \in T}\left\|f(t)-\left(\sum_{j=1}^{n} a_{j} x_{j}\right)^{\tilde{}}(t)\right\| \leq \epsilon .
$$

Now let $\Psi_{0}: Q / \operatorname{ker}(\Psi) \rightarrow \mathbb{B}$ be the induced homomorphism. Then we have $\left\|f-\Psi_{0}\left(\sum_{j=1}^{n} a_{j} x_{j}+\operatorname{ker}(\Psi)\right)\right\| \leq \epsilon$. Thus we have proved that for any $n$ there is an element $y_{n} \in Q / \operatorname{ker}(\Psi)$ such that $\left\|f-\Psi_{0}\left(y_{n}\right)\right\| \leq 1 / n$. Since $\Psi_{0}$ is isometric, $\left(y_{n}\right)_{n}$ is a Cauchy sequence and hence convergent to an element $y \in$ $Q / I$. Obviously $f=\Psi_{0}(y)$, and so for any preimage $x \in \mathbb{Q}$ of $y$ we will have $f=\tilde{x}$.

(iv) is trivial.

This representation $\Psi$ may be rather trivial. In fact, by $[11,7.16]$ there is a separable type-I $\mathrm{C}^{*}$-algebra with a rich ideal structure such that the center is trivial (a unit should be adjoined to this example). But things are much better in the liminal case, as seen in the following.

Theorem 5.6. Let $Q$ be a liminal separable unital $C^{*}$-algebra. Then the representation $\Psi$ described above is faithful. There is an open dense subset 
$U \subset T$ such that all functions $\|\tilde{x}(\cdot)\|$ are continuous on $U$ and such that the fibres $Q(t)$ are elementary for all $z \in U$. Moreover, $\left.\tilde{x}\right|_{U}=0 \Leftrightarrow x=0$.

If $\operatorname{Prim}(\mathfrak{Q})$ is Hausdorff then we may choose $U=T$, and the representation coincides with the representation given in $[12,10.5 .4]$.

Proof. Let $U_{0}$ be the interior of the set of separated points in Prim( $(\mathcal{Q})$. By Corollary 2.3, $\Phi: U_{0} \rightarrow \Phi\left(U_{0}\right)=: U$ is a homeomorphism onto the open dense subset $U \subset T$. Since $\Phi^{-1}: U \rightarrow U_{0}$ is continuous and since $U_{0} \subset \operatorname{MP}(\mathscr{Q})[3$, Prop. 4.5], we have continuity of $\|\tilde{x}(\cdot)\|=\|x+\cdot\| \bullet \psi$.

For $t \in U$ we have $\psi(t) \in \operatorname{Prim}(\mathfrak{Q})$; hence $Q(t)=\mathscr{Q} / \psi(t)$ is elementary.

Clearly $x=0$ implies $\left.\tilde{x}\right|_{U}=0$. Conversely, let $\left.\tilde{x}\right|_{U}=0$. By the formula $\|\tilde{x}(\cdot)\|=\|x+\cdot\| \circ \psi$ and Theorem 2.2 , we have $\|x+I\|=0$ for all $I$ in a dense subset of $\mathrm{MP}(\mathscr{Q})$. Thus $x=0$.

Finally, if $\operatorname{Prim}(Q)$ is Hausdorff then $U_{0}=\operatorname{Prim}(Q)$, which coincides with $T$ by the Dauns-Hofmann theorem. Then $\Phi$ reduces to the identity map and so does $\psi$. In this case, $\Psi$ is clearly the representation described in [12, 10.5.4]. This finishes the proof of the theorem.

REMARKs. If $Q$ is a separable liminal unital $C^{*}$-algebra with Hausdorff spectrum, then $[12,10.5 .4]$ tells us how we should represent $Q$ as an algebra of continuous operator fields on $\operatorname{Prim}(\mathfrak{Q})$ which coincides with the spectrum of the center. If $Q$ does not have Hausdorff spectrum then by Theorem 5.6 we may still have a faithful representation as operator fields living on the spectrum of the center, but continuity holds only on a dense open subset; only Borel measurability is guaranteed. In this sense Theorem 5.6 is a direct generalization of $[12,10.5 .4]$.

This theorem should also be compared with [7, Thm. 3.9], where Busby constructed a representation of a separable unital $C^{*}$-algebra as a continuous prefield (i.e., continuity of $\|x(\cdot)\|$ for all $x \in Q$, but condition (iii) of Thm. 2.1 doesn't hold). The base space he uses is a compactification of the space $U_{0}$ (used in the proof of Theorem 5.6), which need not coincide with the spectrum of the center of $Q$. (Busby uses the embedding $Q \hookrightarrow M(L(Q))$ of $\mathbb{Q}$ into the multiplier algebra of $L(\mathbb{Q})$, where $L(\mathscr{Q})$ is the ideal of $\mathbb{Q}$ belonging to $U_{0}$.) The representation given here is better adjusted to the center of $Q$; it uses the spectrum of the center as the base space and it maps the center as it should.

Example. Consider Example 4.12 of [3] and the notation used there. We have

$$
T \cong \mathbf{N} \cup\{\infty\},
$$

where $\Phi: \operatorname{Prim}(Q) \rightarrow T$ is the map $P_{n} \rightarrow n$ and $\left\{Q_{1}, Q_{2}, Q_{3}\right\} \rightarrow \infty$. We have that $\operatorname{MP}(\mathscr{Q})=\left\{P_{n} \mid n \in \mathrm{N}\right\} \cup\left\{Q_{1} \cap Q_{2}, Q_{1} \cap Q_{3}, Q_{2} \cap Q_{3}\right\}$ and $\Phi$ maps $P_{n}$ to $n$ and the $Q_{i}$ to $\infty$. So there are three Borel cross-sections $\psi_{i}: T \rightarrow \operatorname{MP}(Q)$, namely $n \rightarrow P_{n}$, and $\infty$ is mapped to one of the minimal primal ideals $Q_{i} \cap Q_{j}$. Observe that none of these is continuous, but in spite of this any central element $x \in Q$ is mapped to a continuous function $\tilde{x}$ on $T$. 


\section{References}

[1] J. F. Aarnes, Physical states on a $C^{*}$-algebra, Acta Math. 122 (1969), 161-172.

[2] —, Quasistates on $C^{*}$-algebras, Trans. Amer. Math. Soc. 149 (1970), 601625.

[3] R. J. Archbold, Topologies for primal ideals, J. London Math. Soc. (2) 36 (1987), 524-542.

[4] - Density theorems for the centre of a $C^{*}$-algebra, J. London Math. Soc. (2) 10 (1975), 189-197.

[5] R. J. Archbold and C. J. K. Batty, On factorial states of operator algebras III, J. Operator Theory 15 (1986), 53-81.

[6] B. Blackadar and D. Handelman, Dimension functions and traces on $C^{*}$-algebras, J. Funct. Anal. 45 (1982), 297-340.

[7] R. C. Busby, On structure spaces and extensions of $C^{*}$-algebras, J. Funct. Anal. 1 (1967), 370-377.

[8] E. Christensen, Measures on projections and physical states, Comm. Math. Phys. 86 (1982), 529-538.

[9] J. Cuntz, Noncommutative Haar measure and algebraic finiteness conditions for simple $C^{*}$-algebras, Colloques Internationaux C.N.R.S. No. 274, Algèbres d'opérateurs et leurs applications en physique mathématique, CNRS, Paris, 1979.

[10] C. Delaroche, Sur les centres des $C^{*}$-algèbres, Bull. Sci. Math. (2) 91 (1967), 105-112.

[11] — , Sur les centres des $C^{*}$-algèbres, II, Bull. Sci. Math. (2) 92 (1968), 111128.

[12] J. Dixmier, $C^{*}$-algebras, North-Holland, Amsterdam, 1977.

[13] — Sur les espaces localement quasi-compact, Canad. J. Math. 20 (1968), 1093-1100.

[14] — Points séparés dans le spectre d'une $C^{*}$-algèbre. Acta Sci. Math. Szeged 22 (1961), 115-128.

[15] E. Effros and F. Hahn, Locally compact transformation groups and $C^{*}$-algebras, Mem. Amer. Math. Soc. 75 (1967).

[16] J. M. G. Fell, A Hausdorff topology for the closed subsets of a locally compact non-Hausdorff space, Proc. Amer. Math. Soc. 13 (1962), 472-476.

[17] G. Gierz, K. H. Hoffmann, K. Keimel, J. D. Lawson, M. Mislove, and D. S. Scott, A compendium of continuous lattices, Springer, Berlin, 1980.

[18] A. Gleason, Measures on the closed subspaces of a Hilbert space, J. Math. Mech. 6 (1957), 885-893.

[19] A. Kaplan, Continuous quasi-states on $C^{*}$-algebras, J. Funct. Anal. 86 (1989), 111-126.

[20] G. K. Pedersen, $C^{*}$-algebras and their automorphism groups, Academic Press, New York, 1979.

[21] F. J. Yeadon, Measures on projections in $W^{*}$-algebras of type $I_{1}$, Bull. London Math. Soc. 15 (1983), 139-145.

Westfälische Wilhelms - Universität Münster

Einsteinstraße 62, 48149 Münster

Federal Republic of Germany 